\title{
The design of hydrodynamic water-lubricated step thrust bearings using CFD method
}

\author{
Xiuli Zhang, Zhongwei Yin ${ }^{\mathrm{a}}$, Dan Jiang and Gengyuan GaO \\ School of Mechanical Engineering, Shanghai Jiao Tong University, Shanghai, PR China
}

Received 28 November 2013, Accepted 26 March 2014

\begin{abstract}
Water-lubricated bearings are expected to be widely used because of convenience, green, safe and energy saving. The purpose of this study is to provide references for designing hydrodynamic waterlubricated step thrust bearings. The numerical analysis is undertaken under the condition of different pad dimensions, step heights, step positions, water film thicknesses and rotational speeds of thrust rings based on computational fluid dynamics (CFD). The results including pressure distribution, load carrying capacity, friction torque and friction coefficient are gained and compared for optimizing geometry parameters. A reference to determine water-lubricated step thrust bearing dimensions and a formula to check the minimum water film thickness are proposed.
\end{abstract}

Key words: Step thrust bearings / hydrodynamic lubrication / water / CFD

\section{Introduction}

Step thrust bearings are widely used in pumps, highspeed turbomachineries to support axial loads because of its easy manufacturing and low cost $[1,2]$. Presently the conventional lubricant is oil, but it has many disadvantages such as serious waste of resources, environmental pollution caused by oil leakage, explosive hazard, large bulk and complex structure, poor flexibility and maneuverability [3]. Moreover, oil-lubrication is infeasible in some special condition such as nuclear pumps. Water resources are rich and water has advantages of convenience, green, safe and energy saving, therefore, more and more attention has been paid to water-lubrication [4]. However, water has a lower viscosity than that of oil, and the load carrying capacity of water-lubricated bearings is much smaller than that of oil-lubricated bearings. Works on water-lubricated bearings [5-8] showed that the bearings usually operate in the regime of mixed lubrication and the lubrication mode might convert to hydrodynamic when load decreases. Wang et al. $[5,6]$ studied the critical load, considering the sudden increase in the friction coefficient as the transition of lubrication mode. The present work focuses on the hydrodynamic water-lubrication, considers the minimum water film thickness as a criterion of lubrication mode and aims at giving a reference for designing hydrodynamic water-lubricated step thrust bearings.

${ }^{a}$ Corresponding author: yinzw@sjtu.edu.cn
Step thrust bearings have traditionally been analyzed using Reynolds equation since the early twentieth century. In 1918 Rayleigh [9] analyzed several kinds of slider bearing profiles and found that a step convergence showed in Figure 1a would provide the greatest load per unit width neglecting side leakage. Archibald [10] considered side leakage when studying the load carrying capacity of a square step slider. Kettleborough investigated step thrust bearings with an electrolytic tank in 1953 [11] and by experiments in 1955 [12]. Then Rohde [13] used the finite element method to solve the problem. The optimum variables they gained for designing a straight step bearing are summarized in Table 1 . It indicates that the optimum step height $\delta$ is about 0.7 times of the minimum film thickness $h_{2}$, and the optimum $L_{1} / L_{2}$ changes with pad width-to-length ratio $B / L$. However, the sector pad was simplified to rectangle slider when they did the theoretical derivation or numerical simulation, and oil grooves were ignored, thereby reducing computation. There are also many research works in which infinite width step slider bearing hydrodynamic problems were solved by solving Reynolds Equation using numerical schemes or analytical method [1, 14].

In recent years, with the development of computer technology, many researchers have used commercial CFD programs which are based on the full Navier-Stokes equations to solve lubrication problems. Chen et al. [15] demonstrated the validity of using CFD software to handle hydrodynamic lubrication problems pertaining to slider bearings, step bearings, journal bearings and 


\section{Nomenclature}

\begin{tabular}{|ll|}
\hline$B$ & Pad width $=R_{2}-R_{1}$ \\
$B / L$ & Pad width-to-length ratio \\
$f$ & Friction coefficient $=M_{\mathrm{fr}} /\left(W R_{\mathrm{m}}\right)$ \\
$h_{1}, h_{2}$ & Film thicknesses at inlet and outlet of clearance \\
$h_{\mathrm{g}}$ & Groove depth \\
$k$ & Pad ratio $=\alpha /(\alpha+\beta)$ \\
$L, L_{1}, L_{2}$ & Pad lengths (see Fig. 1$), L=2 \pi R_{\mathrm{m}} k / n=L_{1}+L_{2}$ \\
$L_{\mathrm{g}}$ & Groove length \\
$M_{\mathrm{fr}}$ & Friction torque on the thrust ring \\
$M_{\mathrm{fr}}^{*}$ & Non-dimensional friction torque $=M_{\mathrm{fr}} h_{2} /\left(n \mu U_{\mathrm{m}} L B R_{\mathrm{m}}\right)$ \\
$N$ & Rotational speed \\
$n$ & Number of pads \\
$p$ & Pressure \\
$p^{*}$ & Non-dimensional pressure $=p h_{2}^{2} /(\mu U L)$ \\
$R_{1}, R_{\mathrm{m}}, R_{2}$ & Bearing inner, middle and outer radius \\
$R_{\mathrm{pmax}}$ & Radius of the maximum pressure \\
$R_{\mathrm{pmax}}^{*}$ & Non-dimensional radius of the maximum pressure $=\left(R_{\mathrm{pmax}}-R_{1} / B\right)$ \\
$U$ & Linear velocity of the thrust collar $=2 \pi N R, U_{\mathrm{m}}=2 \pi N R_{\mathrm{m}}$ \\
$\mathbf{v}$ & Velocity vector \\
$W$ & Load carrying capacity of a thrust bearing \\
$W^{*}$ & Non-dimensional load carrying capacity $=W h_{2}^{2} /\left(n \mu U_{\mathrm{m}} L^{2} B\right)$ \\
$\alpha, \beta$ & Pad angle, groove angle \\
$\delta$ & Step height = $h_{1}-h_{2}$ \\
$\delta^{*}$ & Non-dimensional step height $=\delta / h_{2}$ \\
$\mu$ & Fluid dynamic viscosity \\
$\rho$ & Fluid density \\
\hline
\end{tabular}

Table 1. The optimum design of a straight step bearing in the literature.

\begin{tabular}{cccc}
\hline Authors & $B / L$ & Optimum design parameters & $W^{*}$ \\
\hline Rayleigh & $\infty$ & $\delta^{*}=0.87, L_{1} / L_{2}=2.549$ & 0.206 \\
Archibald & 1 & $\delta^{*}=0.70, L_{1} / L_{2}=1.222$ & 0.0725 \\
& 0.5 & $\delta^{*}=0.68, L_{1} / L_{2}=1.041$ & 0.027 \\
Rohde & 1 & $\delta^{*}=0.69, L_{1} / L_{2}=1.222$ & 0.0726 \\
& 2 & $\delta^{*}=0.74, L_{1} / L_{2}=1.778$ & 0.127 \\
& 4 & $\delta^{*}=0.81, L_{1} / L_{2}=2.226$ & 0.165 \\
\hline
\end{tabular}

squeeze-film dampers. Vakilian et al. [2] studied inertia effect on thermohydrodynamic characteristics of Rayleigh step bearings by CFD method. Lo et al. [16] used CFD code to analyze the effects of the rolling speed, oil viscosity, and supplying oil concentration on the pressure distributions of oil and water phases. Zhang et al. [17], Brajdic-Mitidieri et al. [18] and Papadopoulos et al. [19] optimized the geometry of inclined thrust bearings, pocketed pad bearings and textured micro-thrust bearings using CFD techniques respectively. CFD widens the range of solvable lubrication problems and makes design process more convenient.

The present work investigates the step thrust bearings with sector pads and straight radial grooves under hydrodynamic lubrication by water. Three-dimensional CFD models with different bearing dimensions, film thicknesses, step heights and positions were computed to study the effects of them on load carrying capacities, friction torques and friction coefficients.

\section{Step thrust bearing model}

Figure 1b shows the three-dimensional flow model of a sector pad and its groove. The thrust bearing is submersed in water. The hydrodynamic action generates dynamic pressure in water, primarily in the convergent part of the thrust pad, to counteract the load thereby separating the ring surface from the bearing surface with a thin lubricant film. Geometry parameters - inner radius $R_{1}$, outer radius $R_{2}$, number of pads $n$, pad ratio $k$, step height $\delta$ and step position $L_{1} / L_{2}-$ all influence the load carrying capacity of step thrust bearings. According to the design of oil-lubricated thrust bearings [20], $R_{2}$ is usually $1.5-3$ times of $R_{1}$. The number of pads $n$ is generally 6 to 12 . The pad ratio $k$, the percentage of pad area in the whole thrust surface, is typically $0.7-0.85$. Pad width-to-length ratio $B / L$ is a pad parameter determined by $R_{1} / R_{2}, n$ and $k$, and the relation is

$$
\frac{B}{L}=\frac{n}{\pi k} \frac{R_{2} / R_{1}-1}{R_{2} R_{1}+1}
$$

It has an effect on the optimum value of the step height $\delta$ and step position $L_{1} / L_{2}$.

Rotational speed $N$, fluid viscosity $\mu$ and the minimum film thickness $h_{2}$ determine the bearing carrying performance as well. Rotational speed and fluid viscosity are determined by work conditions. The minimum film thickness should not be less than a safety value depending on surface roughness and system vibration, or the lubrication mode might change from full dynamic lubrication to boundary or mixed lubrication. 


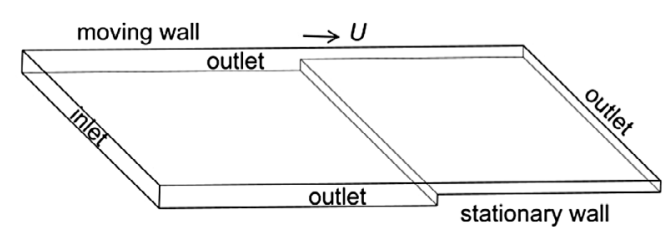

(a)

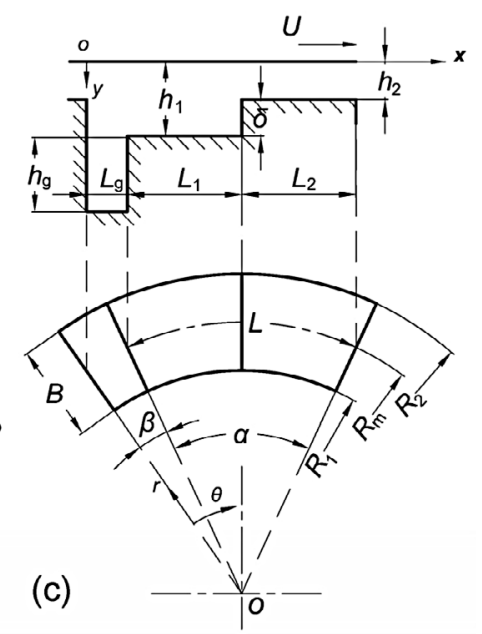

Fig. 1. (a) Three-dimensional flow model of a rectangular pad (not to scale) which is studied in the literature. (b) Threedimensional flow model of a sector pad and its groove (not to scale). (c) Geometry parameters of model in (b). (Stationary wall is the thrust pad and moving wall is the thrust ring.)

\section{Numerical analysis}

\subsection{Governing equations}

The flow is considered laminar isothermal, steady and incompressible, with zero gravitational and other external body forces. The bearing is fully submerged into water. To solve such flow, the following governing equations must be solved:

Mass conservation equation

$$
\nabla \cdot \mathbf{v}=0
$$

Momentum conservation equations

$$
\rho(\mathbf{v} \cdot \nabla) \mathbf{v}=-\nabla p+\mu \cdot \nabla^{2} \mathbf{v}
$$

When flow enters the groove, pressure might fall below the saturation water vapor pressure, and the liquid would rupture and cavitation occurs. Thus cavitation is taken into account. In the present work the CFD code ANSYS FLUENT is used. There are three available cavitation models in ANSYS FLUENT: Singhal et al. model, Zwart-Gerber-Belamri model and Schnerr and Sauer model. The Singhal et al. model is numerically less stable and more difficult to use. The Zwart-Gerber-Belamri and the Schnerr and Sauer models are robust and converge quickly [21]. In this case CFD models with the latter two cavitation models are calculated when studying the mesh refinement. Table 6 shows that the Zwart-GerberBelamri model is less sensitive to mesh density. Thus the Zwart-Gerber-Belamri model is employed.

In cavitation, the liquid-vapor mass transfer (evaporation and condensation) is governed by the vapor transport equation [21]:

$$
\frac{\partial}{\partial t}\left(\alpha_{\mathrm{v}} \rho_{\mathrm{v}}\right)+\nabla \cdot\left(\alpha_{\mathrm{v}} \rho_{\mathrm{v}} \mathbf{v}\right)=R_{\mathrm{g}}-R_{\mathrm{c}}
$$

where $R_{\mathrm{g}}$ and $R_{\mathrm{c}}$ account for the mass transfer source terms connected to the growth and collapse of the vapor bubbles, $\alpha_{\mathrm{v}}$ is vapor volume fraction and $\rho_{\mathrm{v}}$ is vapor density. In Zwart-Gerber-Belamri model, $R_{\mathrm{g}}$ and $R_{\mathrm{c}}$ are defined as follows [22]:

$$
\begin{aligned}
& \text { If } p \leqslant p_{\mathrm{v}} R_{\mathrm{g}}=F_{\text {evap }} \frac{3 \alpha_{\mathrm{nuc}}\left(1-\alpha_{\mathrm{v}}\right) \rho_{\mathrm{v}}}{R_{\mathrm{b}}} \sqrt{\frac{2}{3} \frac{p_{\mathrm{v}}-p}{\rho_{\mathrm{l}}}} \\
& \text { If } p \geqslant p_{\mathrm{v}} R_{\mathrm{c}}=F_{\text {cond }} \frac{3 \alpha_{\mathrm{v}} \rho_{\mathrm{v}}}{R_{\mathrm{b}}} \sqrt{\frac{2}{3} \frac{p-p_{\mathrm{v}}}{\rho_{\mathrm{l}}}}
\end{aligned}
$$

where $F_{\text {evap }}=$ evaporation coefficient $=50, F_{\text {cond }}=$ condensation coefficient $=0.01, R_{\mathrm{b}}=$ bubble radius $=$ $10^{-6} \mathrm{~m}, \alpha_{\text {nuc }}=$ nucleation site volume fraction $=5 \times$ $10^{-4}, \rho_{\mathrm{l}}=$ liquid density, $p_{\mathrm{v}}=$ pressure of vapor.

Supposed that there's no installation error and the load of a thrust bearing is distributed uniformly over all pads, the load and friction torque of a thrust bearing can be calculated by integrating the pressure and shear stress over the rotating wall as follows:

$$
W=n \int_{R_{1}}^{R_{2}} \int_{0}^{\alpha+\beta} p r \mathrm{~d} \theta \mathrm{d} r, M_{\mathrm{fr}}=n \int_{R_{1}}^{R_{2}} \int_{0}^{\alpha+\beta} \tau r^{2} \mathrm{~d} \theta \mathrm{d} r
$$

The friction coefficient is

$$
f=\frac{M_{\mathrm{fr}}}{W R_{\mathrm{m}}}
$$

To compare with results in the literature, nondimensional pressure, non-dimensional load carrying capacity and non-dimensional friction torque are defined as

$$
p^{*}=\frac{p h_{2}^{2}}{\mu U L}, W^{*}=\frac{W h_{2}^{2}}{n \mu U_{\mathrm{m}} L^{2} B}, M_{\mathrm{fr}}^{*}=\frac{M_{\mathrm{fr}} h_{2}}{n \mu U_{\mathrm{m}} L B R_{\mathrm{m}}}
$$

\subsection{Boundary conditions}

The boundary condition is set as shown in Figure 1b. Rotational periodic boundary condition is used to simplify the flow model and to reduce the computational 
Table 2. Water properties at $20{ }^{\circ} \mathrm{C}$.

\begin{tabular}{lc}
\hline Saturation water vapor pressure & $2340 \mathrm{~Pa}$ \\
Saturation density of water & $998.2 \mathrm{~kg} \cdot \mathrm{m}^{-3}$ \\
Saturation density of water vapor & $0.5542 \mathrm{~kg} \cdot \mathrm{m}^{-3}$ \\
Dynamic viscosity of water & $10^{-3} \mathrm{~Pa} . \mathrm{s}$ \\
Dynamic viscosity of water vapor & $1.34 \times 10^{-5} \mathrm{~Pa} . \mathrm{s}$ \\
\hline
\end{tabular}

cost. The operating pressure is set to 101325 Pa. Since the bearing is fully submerged, the pressure at the inlet and outlet boundaries is taken as zero (gauge pressure). A no-slip condition is imposed on the solid walls. The bottom wall is stationary and the upper one is assumed to be rotating at a constant rotational speed $N$.

\subsection{CFD models}

In the present study, water properties at $20{ }^{\circ} \mathrm{C}$ listed in Table 2 are employed. In all cases, the groove depth $h_{\mathrm{g}}=2 \mathrm{~mm}$ and pad ratio $k=0.75$. For the purpose of finding relations between $B / L, \delta$ and $L_{1} / L_{2}$, the inner radius $R_{1}$ is set to $30 \mathrm{~mm}$, number of pads $n$ is 12 , rotational speed $N$ is $3000 \mathrm{r} \cdot \mathrm{min}^{-1}$ and $R_{2}, \delta^{*}, L_{1} / L_{2}$ vary as shown in Table 3 . The non-dimensional step height $\delta^{*}$ and the step position $L_{1} / L_{2}$ vary to seek out the optimum ones. The outer radius $R_{2}$ changes to find the effect of $B / L$ on the non-dimensional load $W^{*}$, the optimum $\delta^{*}$ and the optimum $L_{1} / L_{2}$. Then models with different sizes and rotational speeds are analyzed to get design reference for bearing dimensions. Inner radius $R_{1}$ ranges from $5 \mathrm{~mm}$ to $40 \mathrm{~mm}$ and outer radius $R_{2}$ is 1.5 times, twice and 3 times $R_{1}$, respectively. The number of pads varies as shown in Table 4 . Pad width-to-length ratio $B / L$ can be calculated from equation (1). The step height $\delta$ and the step position $L_{1} / L_{2}$ are the optimum values gained from the study above. The minimum film thicknesses increase as the bearings get larger to ensure that the bearings operate under hydrodynamic lubrication. The values are shown in Table 5. The rotational speeds range from 100 r.min ${ }^{-1}$ to 5000 r.min ${ }^{-1}$.

Due to the existence of the thin film in hydrodynamic bearings, the dominating feature for bearing CFD models is the large aspect ratio of the grid which is $330 \sim 4500$ in this study. However, the suggested value of aspect ratio is less than 100 or 200 for normal analysis. Thus double precision calculations are employed to avoid the negative influence of the large aspect ratio according to ANSYS FLUENT user manual [21]. The CFD models are meshed using hexahedron grids in Gambit 2.3. In order to obtain accurate solutions, a mesh refinement study is carried out. Since the groove area contributes little to the load carrying capacity, the groove length $L_{\mathrm{g}}$ is divided into 15 cells and the groove height $h_{\mathrm{g}}$ is divided into 20 cells for all cases. An expansion ratio of 1.4 is employed in $h_{\mathrm{g}}$ to avoid sudden change of mesh density near the thin film thickness. A uniform mesh is employed in other edges of the model. The influence of the mesh density of $h_{2}, \delta, L_{1}$, $L_{2}, B$ on load for the bearing model with $R_{1}=30 \mathrm{~mm}$,
$R_{2}=45 \mathrm{~mm}, n=12, h_{2}=10 \mu \mathrm{m}, \delta=7 \mu \mathrm{m}, L_{1} / L_{2}=1$, $N=3000 \mathrm{r} \cdot \mathrm{min}^{-1}$ is studied and the results are summarized in Table 6 . When the Zwart-Gerber-Belamri model is used, the relative difference in load between case 6 and case 9 is only $0.2 \%$. Based on this, case 6 is employed in this study. Appropriate mesh sizes are also found for other sizes of bearing models.

\section{Results and discussion}

\subsection{Pressure distribution}

Figures 2a-2d present the gauge pressure distribution on the pad surface (the stationary wall) for pads with $\delta^{*}=0.7, L_{1} / L_{2}=1.25, B / L=1.02$ and pressure distribution on the ring surface (the rotating wall) for pads with different $B / L$ values. Pressure distribution for pads with $B / L=4.00$ is presented here for comparison. Figures $2 \mathrm{a}-2 \mathrm{~b}$ show that the maximum pressure of the whole film is located at the step of the pad. Figures $2 \mathrm{~b}-2 \mathrm{~d}$ illustrate that as $B / L$ increases, the pressure center moves towards the outer radius, where the fluid velocity is much larger than that at the middle radius. Non-dimensional radii of the maximum pressure $R_{p_{\max }^{*}}^{*}$, for pads with $B / L=1.02,1.70,4.00$ are $0.537,0.62$ and 0.78 respectively. Figure 3 presents the distribution of $p^{*}$ at $R_{p_{\max }}$ in Figures $2 \mathrm{a}-2 \mathrm{~d}$. Comparing the three solid lines, it is found that the maximum $p^{*}$ on the rotating wall rises from 0.244 to 0.305 as $B / L$ increases from 1.02 to 1.70 , but only increases by 0.007 as $B / L$ increases from 1.70 to 4.00 . It indicates that as the pressure center moves outwards, the pressure buildup is still affected by side leakage for pads with large $B / L$ values. The line " $B / L=1.02 \mathrm{sw}$ " shows that at the step $\left(\theta=20^{\circ}\right)$ and at the entrance to the thin film from the groove $\left(\theta=7.5^{\circ}\right)$ pressure on the stationary wall has a sudden increase, which is known as the "ram effect" [23]. When the fluid flows into the groove $\left(\theta=0^{\circ}, \theta=30^{\circ}\right)$, pressure on the stationary wall drops rapidly, which might cause cavitation if it is less than $2340 \mathrm{~Pa}$, i.e. gauge pressure $98985 \mathrm{~Pa}$. The vapor fraction distribution of the model in Figure 2a is shown in Figure 4. As the fluid velocity near the outer radius is larger than that near the inner radius, the pressure drop is more significant and cavitation occurs more easily. From the CFD analyses, it is found that the cavitation zone will get larger when the minimum film thickness decreases or the fluid velocity increases.

\subsection{Effect of step height}

Figure 5 presents the non-dimensional load carrying capacity $W^{*}$ versus the non-dimensional pad height $\delta^{*}$ for a range of $h_{2}$ from $5 \mu \mathrm{m}$ to $10 \mu \mathrm{m}$ with $B / L=1.02$ and $L_{1} / L_{2}=1.25$. Results illustrate that although the optimum step height is different for different minimum film thicknesses, the optimum ratio of them, the nondimensional pad height $\delta^{*}$, is always around 0.67 , similar 
Table 3. Values of variable parameters.

\begin{tabular}{lccc}
\hline$R_{2}(\mathrm{~mm})$ & $B / L$ & $\delta^{*}$ & $L_{1} / L_{2}$ \\
\hline $35,40,45,50,55,60,65,70$, & $0.39,0.73,1.02,1.27,1.50,1.70,1.88$, & $0.3-1.2$ & $0.75,1,1.25,1.5,1.75,2$, \\
$80,90,100,125,175,25$ & $2.04,2.31,2.55,2.74,3.12,3.60,4.00$ & & $2.25,2.5,2.75,3$ \\
\hline
\end{tabular}

Table 4. Number of pads for different bearing dimensions.

\begin{tabular}{|c|c|c|c|c|c|c|c|c|c|c|c|c|c|c|c|}
\hline $\mathrm{R}_{2} / \mathrm{R}_{1} \mathrm{R}_{1}$ & 5 & 7.5 & 10 & 12.5 & 15 & 17.5 & 20 & 22.5 & 25 & 27.5 & 30 & 32.5 & 35 & 37.5 & 40 \\
\hline 1.5 & & 6 & & 8 & \multicolumn{4}{|c|}{10} & \multicolumn{7}{|c|}{12} \\
\hline 2 & \multicolumn{4}{|c|}{6} & \multicolumn{3}{|c|}{8} & \multicolumn{3}{|c|}{10} & \multicolumn{5}{|c|}{12} \\
\hline 3 & \multicolumn{4}{|c|}{6} & \multicolumn{4}{|c|}{8} & \multicolumn{4}{|c|}{10} & \multicolumn{3}{|c|}{12} \\
\hline
\end{tabular}

Table 5. The minimum film thicknesses.

\begin{tabular}{ccccccccc}
\hline$R_{1}(\mathrm{~mm})$ & 5 & 10 & 15 & 20 & 25 & 30 & 35 & 40 \\
\hline$h_{2}(\mu \mathrm{m})$ & 10 & 12 & 14 & 16 & 18 & 20 & 22 & 24 \\
\hline
\end{tabular}

Table 6. Mesh refinement study.

\begin{tabular}{|c|c|c|c|c|c|c|}
\hline \multirow{2}{*}{ Case } & \multicolumn{3}{|c|}{ Number of cells } & \multirow{2}{*}{ Number of hexahedral elements } & \multicolumn{2}{|c|}{$W(N)$} \\
\hline & $h_{2}(\delta)$ & $L_{1}\left(L_{2}\right)$ & $B$ & & Schnerr and Sauer model & Zwart-Gerber-Belamri model \\
\hline 1 & 6 & 23 & 45 & 40230 & 102.71 & 107.64 \\
\hline 2 & 8 & 23 & 45 & 49140 & 101.82 & 108.9 \\
\hline 3 & 10 & 23 & 45 & 58050 & 99.85 & 108.23 \\
\hline 4 & 12 & 23 & 45 & 66960 & 97.92 & 108.7 \\
\hline 5 & 10 & 30 & 45 & 67500 & 101.73 & 109.76 \\
\hline 6 & 10 & 30 & 50 & 75000 & 104.3 & 110.27 \\
\hline 7 & 10 & 4 & 5 & 90000 & 106.34 & 110.27 \\
\hline 8 & 10 & 50 & 60 & 126000 & 107.71 & 110.28 \\
\hline 9 & 10 & 100 & 100 & 360000 & 109.9 & 110.52 \\
\hline \multicolumn{5}{|c|}{ Maximum variation of $W(\%)$} & 10.9 & 2.6 \\
\hline
\end{tabular}

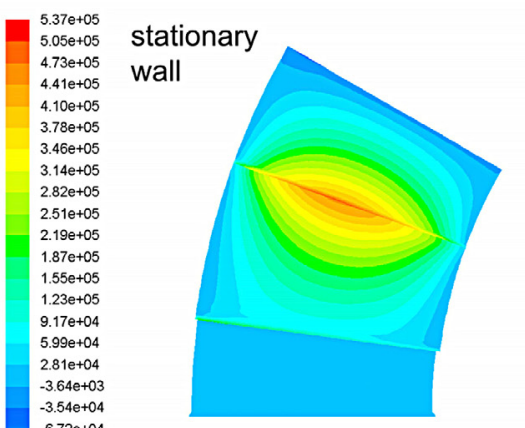

(a) $B / L=1.02$

$1.05 e+06$
$9.97 e+05$
$939+05$

$9.97 \mathrm{e}+05$
$9.39 \mathrm{e}+05$
$8.82 e+05$

$8.82 \mathrm{e}+05$

$7.24 \mathrm{e}+05$

$7.609+05$

$7.09 e+05$
$6.51 \mathrm{e}+05$

$6.516+05$
$5.93+05$

$5.36 \mathrm{e}+05$

$4.78 \mathrm{e}+05$

$4.200+05$

$3.62 \mathrm{e}+05$

$3.05 \mathrm{e}+05$

$2.47 \mathrm{e}+05$

$1.89+05$

$1.32 \mathrm{e}+05$

$7.41 \mathrm{e}+04$

$1.64 \mathrm{e}+04$

$-4.13 e+04$

$00+04$

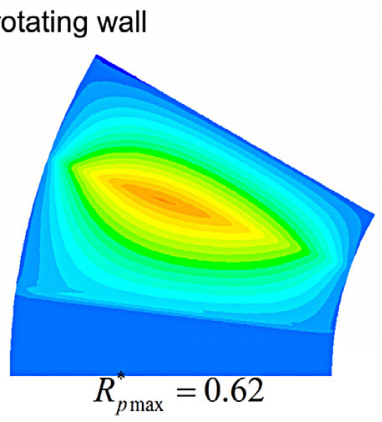

(c) $B / L=1.70$

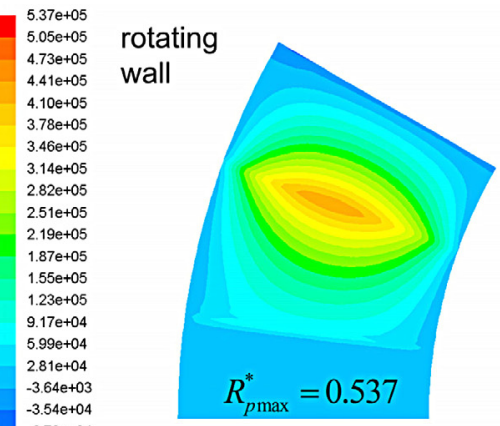

(b) $B / L=1.02$

rotating wall

$1.76 e+07$

$1.67 e+07$
$1.57 e+07$

$1.57 e+07$
$1.48+07$

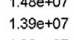

$1.300+07$

$1.20 \mathrm{e}+07$

$1.02 \mathrm{e}+07$

$9.22 \mathrm{e}+06$

$8.29 \mathrm{e}+06$

$7.36 \mathrm{e}+06$

$5.49 \mathrm{e}+06$

$4.56 \mathrm{e}+06$

$3.63 \mathrm{e}+06$

$2.70 \mathrm{e}+06$

$1.77 \mathrm{e}+06$

$-9.900+04$

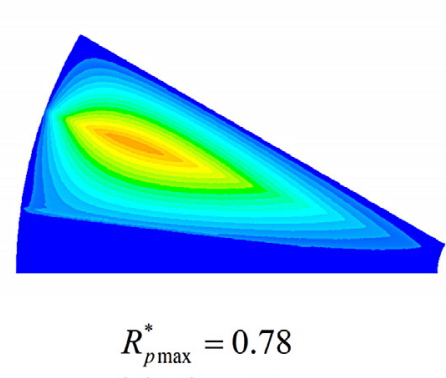

(d) $B / L=4.00$

Fig. 2. Pressure distribution (a) on the stationary wall for pads with $B / L=1.02$, and pressure distribution on the rotating wall for pads with (b) $B / L=1.02$, (c) $B / L=1.70$, (d) $B / L=4.00$, and $\delta^{*}=0.7, L_{1} / L_{2}=1.25, h_{2}=10 \mu \mathrm{m}$. 


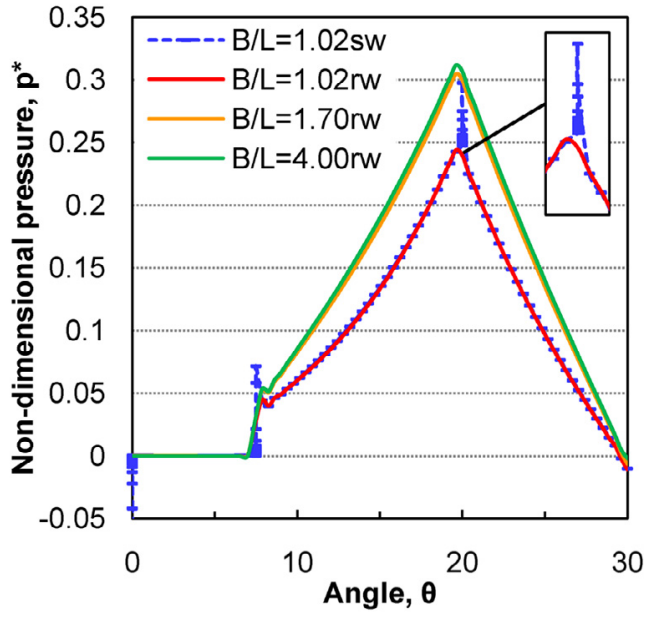

Fig. 3. Non-dimensional pressure distribution at the radius of the maximum pressure in Figures 2a-2d ("sw" refers to the stationary wall and "rw" refers to the rotating wall).
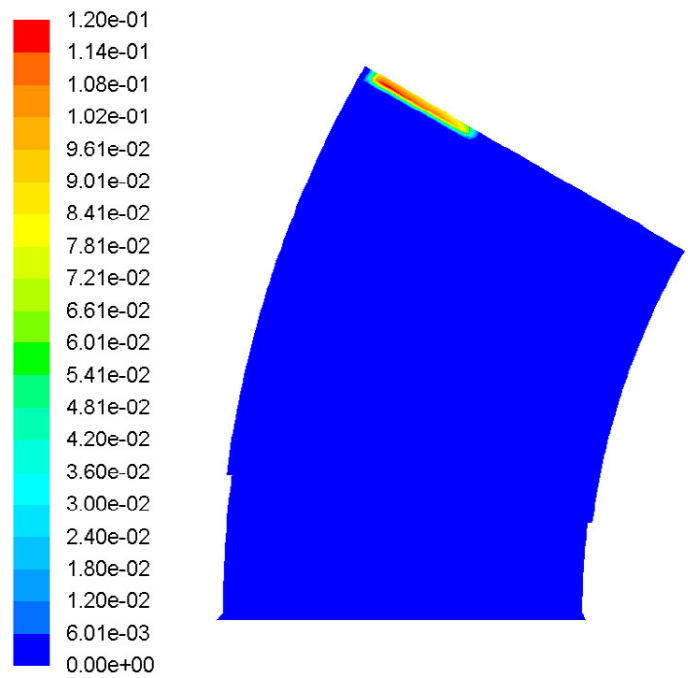

Fig. 4. Vapor fraction distribution of the model in Figure 2a.

to the solutions of Archibald and Rohde. Figure 6 compares the optimum non-dimensional step height $\delta^{*}$ for pads with different $B / L$ values. It can be seen that the optimum $\delta^{*}$ is 0.67 for $B / L=0.73,0.68$ for $B / L=1.27$, 0.69 for $B / L=2.31,3.12$ and 4.00 . That means that $B / L$ has a little effect on the optimum $\delta^{*}$, which is different from Rohde's results (see Tab. 1). This is because in a rotating sector water film, side leakage is still prominent for large values of $B / L$ due to centrifugal force [24]. Table 7 compares the side water mass flow rate (at the inlet and outlet boundaries) of a sector flow model and a rectangular flow model which has the same $B, L_{\mathrm{g}}, L_{1}, L_{2}$ as that of the sector model for different $B / L$ values. The side water mass flow rate of a sector film is almost 10-fold that of a rectangular film. Centrifugal force in a rotating sector water film increases the side mass flow rate and diminishes the effect of $B / L$ on the optimum $\delta^{*}$. The trends in Figures 5 and 6 also show that any change of $\delta^{*}$ results in the decrease of $W^{*}$, which is over 8 percent when $\delta^{*}$

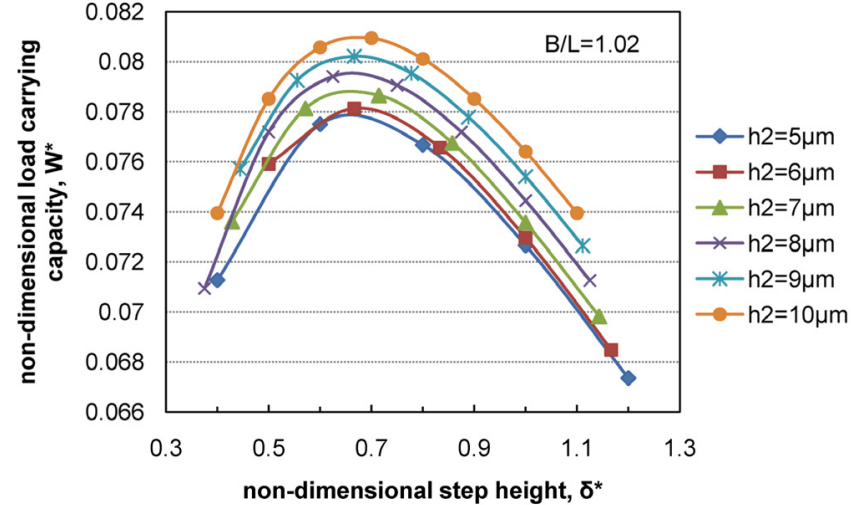

Fig. 5. Non-dimensional load carrying capacity $W^{*}$, versus non-dimensional pad height, $\delta^{*}$, for different film thicknesses, $h_{2}$ and $B / L=1, L_{1} / L_{2}=1.25$.

Table 7. Side mass flow rate of a sector flow model compared with that of a rectangular flow model for pads with different $B / L$ values (Unit: $\mathrm{kg} . \mathrm{s}^{-1}$ ).

\begin{tabular}{ccc}
\hline$B / L$ & Sector model & Rectangular model \\
\hline 0.73 & 0.00064 & 0.00006 \\
1.27 & 0.0017 & 0.00017 \\
2.31 & 0.0066 & 0.00064 \\
3.12 & 0.016 & 0.0012 \\
4 & 0.04 & 0.0023 \\
\hline
\end{tabular}

is less than 0.4 or more than 1.1 , but less than 1 percent when $\delta^{*}$ is between 0.6 and 0.8 .

Figures $7 \mathrm{a}-7 \mathrm{f}$ present the influence of non-dimensional pad height $\delta^{*}$ on non-dimensional friction torque $M_{\mathrm{fr}}^{*}$ and friction coefficient $f$ for pads with $B / L=0.39,1.02$ and 3.12 respectively. As the step height increases, $M_{\mathrm{fr}}^{*}$ decreases monotonically, and $f$ first decreases and then increases with a minimum at $\delta^{*}=0.8$. The results follow the same trend for different $B / L$ values. Figures $7 \mathrm{~b}, 7 \mathrm{~d}, 7 \mathrm{f}$ also show that $f$ decreases by almost $25 \%$ from $\delta^{*}=0.3$ to $\delta^{*}=0.8$, but it varies slightly when $\delta^{*}$ is in the range $[0.6$, 1.0]. Thus considering synthetically the load carrying capacity and friction performance, the step height can be designed as follows:

$$
\delta=(0.6 \sim 0.8) h_{2}
$$

\subsection{Effect of step position}

Figure 8 presents the dependence of non-dimensional load carrying capacity on step position, i.e. $L_{1} / L_{2}$, for pads with different widths and $\delta^{*}=0.7$. Similar to the relation of $W^{*}$ with $\delta^{*}$, there is an optimum $L_{1} / L_{2}$ maximizing $W^{*}$. It can be seen that the values of the optimum $L_{1} / L_{2}$ vary with $B / L$. Figure 9 illustrates that the optimum $L_{1} / L_{2}$ resembles a sigmoid curve with the pad width-to-length ratio $B / L$. It is about 1.1 for small width pads $(B / L<1)$ and 1.85 for large width pads $(B / L>3)$. For a range of $B / L[1,2]$ which is commonly used in design, the optimum $L_{1} / L_{2}$ increases rapidly from 1.2 

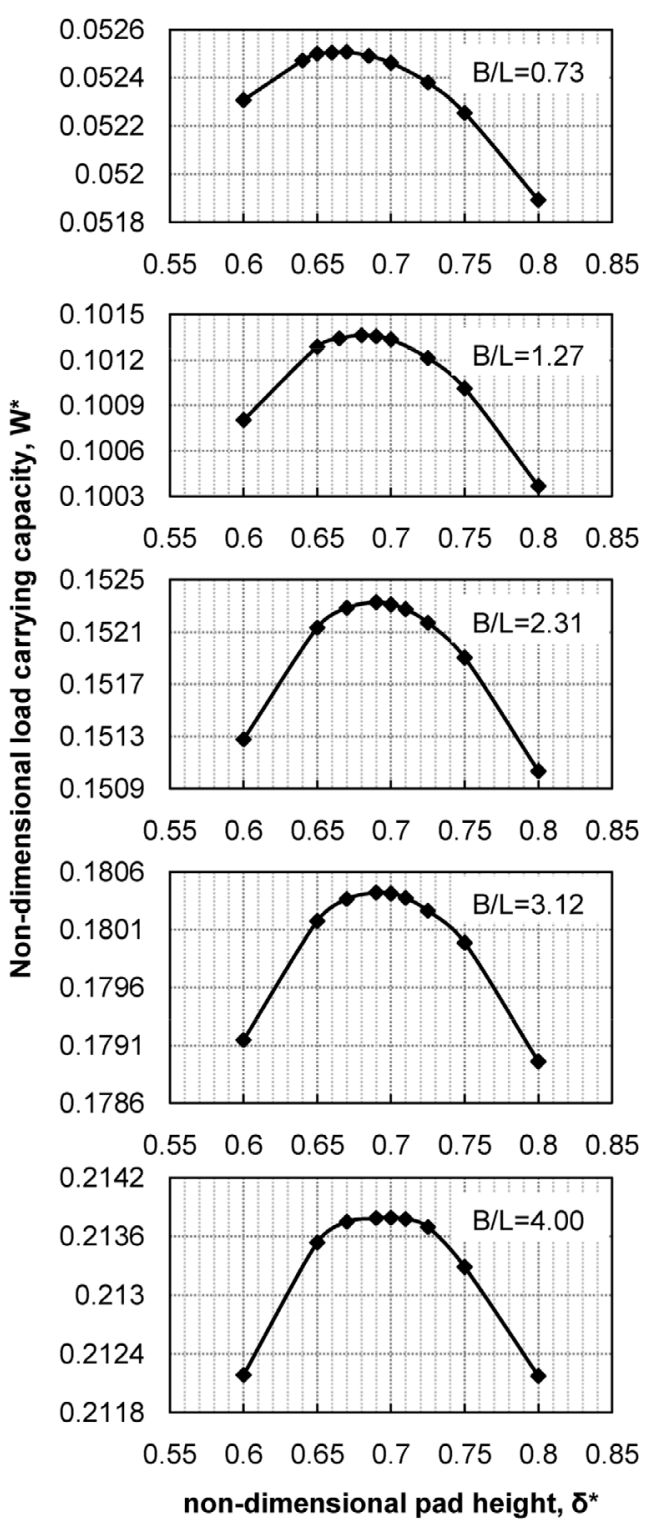

Fig. 6. Non-dimensional load carrying capacity, $W^{*}$, versus non-dimensional pad height, $\delta^{*}$, for pads with different $B / L$ values, and $L_{1} / L_{2}=1.25, h_{2}=10 \mu \mathrm{m}$.

to 1.75. For large width pads, the optimum $L_{1} / L_{2}$ is smaller than Rohde's solution (see Tab. 1), which can also be explained by the centrifugal effect in the rotating sector film.

Figures $10 \mathrm{a}-10 \mathrm{~b}$ present non-dimensional friction torque $M_{\mathrm{fr}}^{*}$ and friction coefficient $f$ versus $L_{1} / L_{2}$ with $B / L=1.02$ respectively. Compared with Figure 7 , the curves have the same trend. Nevertheless, the step position has a larger effect on friction torque, which decreases by about $14 \%$ with $L_{1} / L_{2}$ increasing from 0.75 to 3 , as shown in Figure 10a. The friction coefficient gets the minimum value at $L_{1} / L_{2}=1.75-2$, about $0.55-0.8$ larger than the value of $L_{1} / L_{2}$ at the maximum load, but $f$ changes very small from $L_{1} / L_{2}$ at the maximum load to $L_{1} / L_{2}$ at the minimum friction torque. The same rule is found when $B / L$ is equal to other values. So the nondimensional load carrying capacity can be taken as the principle objective function and $L_{1} / L_{2}$ can be designed according to Figure 9.

\subsection{Selection of bearing diameters}

Figures 11-13 show a design reference obtained by calculating numerical models with different dimensions and rotational speeds. The number on the curve is the load carrying capacity. The outer diameter is 1.5 times, twice and 3 times the inner diameter, respectively. When the load and rotational speed are defined, the bearing inner diameter can be selected from the graphs. For example, as shown in Figures 11-13 (the dash-dotted line), when the rotational speed is $3500 \mathrm{r} . \mathrm{min}^{-1}$ and $200 \mathrm{~N}$ is required for the load carrying capacity, the inner diameter should be over $72.5 \mathrm{~mm}$ when $D_{2}=1.5 D_{1}, 40 \mathrm{~mm}$ when $D_{2}=2 D_{1}$ or $22 \mathrm{~mm}$ when $D_{2}=3 D_{1}$. Designers could determine the bearing diameters according to the mounting dimensions. Figures 11-13 can also be used to check if a specified bearing can meet the load requirements. This is a rough design of bearing diameters. Designers should check the minimum film thickness for further determination.

\subsection{Effect of pad width-to-length ratio and check computation of the minimum film thickness}

Figures $14 \mathrm{a}-14 \mathrm{c}$ present the obtained non-dimensional load carrying capacity $W^{*}$, non-dimensional friction torque $M_{\mathrm{fr}}^{*}$ and friction coefficient $f$, versus pad widthto-length ratio $B / L$ for $\delta^{*}=0.7$ and $L_{1} / L_{2}=1.25$, respectively. As $B / L$ increases from 0.39 to $4, W^{*}$ grows more than 10 -fold, from 0.018 to 0.214 , while $M_{\text {fr }}^{*}$ only increases by $70 \%$. The friction coefficient sharply decreases from 0.037 to 0.008 when $B / L$ increases from 0.39 to 1 , and then gradual decreases to 0.001 . The slope of the load curve is 0.1 for small values of $B / L(<1)$, while reduces to 0.034 for large values of $B / L(>2.5)$. This is because for small $B / L$ values, the pressure buildup is more affected by side leakage. $W^{*}$ for $B / L=0.5,1,2,4$ is larger than that obtained by Rohde, and $W^{*}$ for $B / L=4$ is even larger than Rayleigh's solution for infinite width sliders, $W^{*}=0.206$. It indicates that sector pads provide greater load carrying capacity than rectangular ones. This can be explained by the pressure distributions for pads with different widths, which is presented in Figure 2. As $B / L$ increases, pressure center in a sector pad moves towards the outside radius, where the fluid velocity is much larger than that at the middle radius. Thus larger pressure and greater load are gained in a sector pad than those in a rectangular pad.

The minimum film thickness $h_{2}$ can be calculated from $W^{*}$. As illustrated in Figure $14 \mathrm{a}, B / L$ is the main influence factor on the non-dimensional load carrying capacity $W^{*}$ for a constant non-dimensional step height $\delta^{*}$ and step position $L_{1} / L_{2}$. This means for a certain $B / L$ value, $W^{*}$ is definite. Then the minimum film thickness 

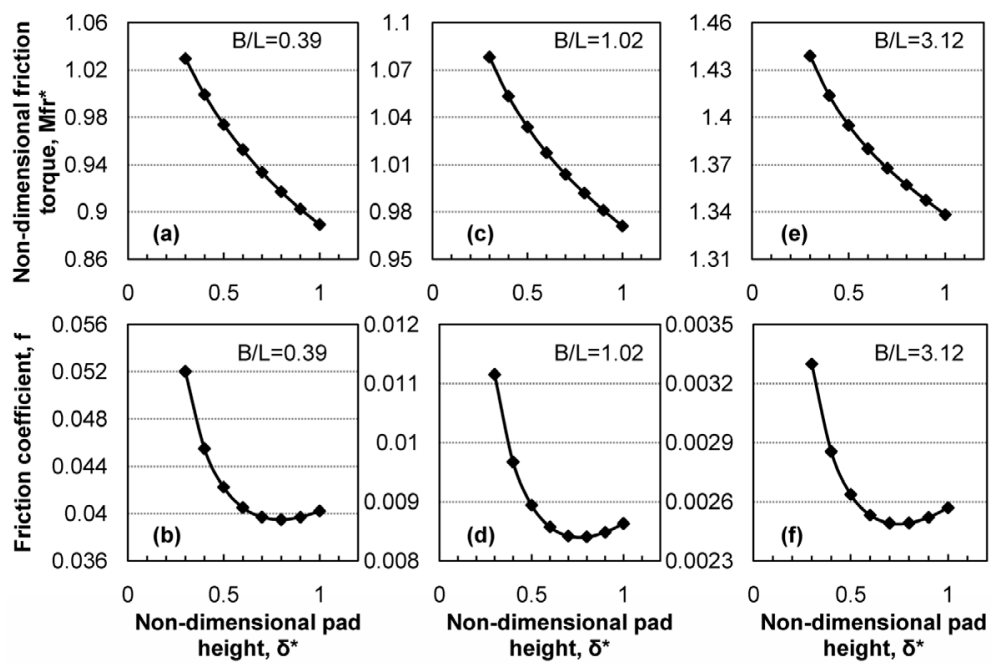

Fig. 7. Non-dimensional friction torque, $M_{\mathrm{fr}}^{*}$, and friction coefficient, $f$, versus non-dimensional pad height, $\delta^{*}$, for pads with $B / L=0.39,1.02$ and $3.12, L_{1} / L_{2}=1.25$ and $h_{2}=10 \mu \mathrm{m}$.
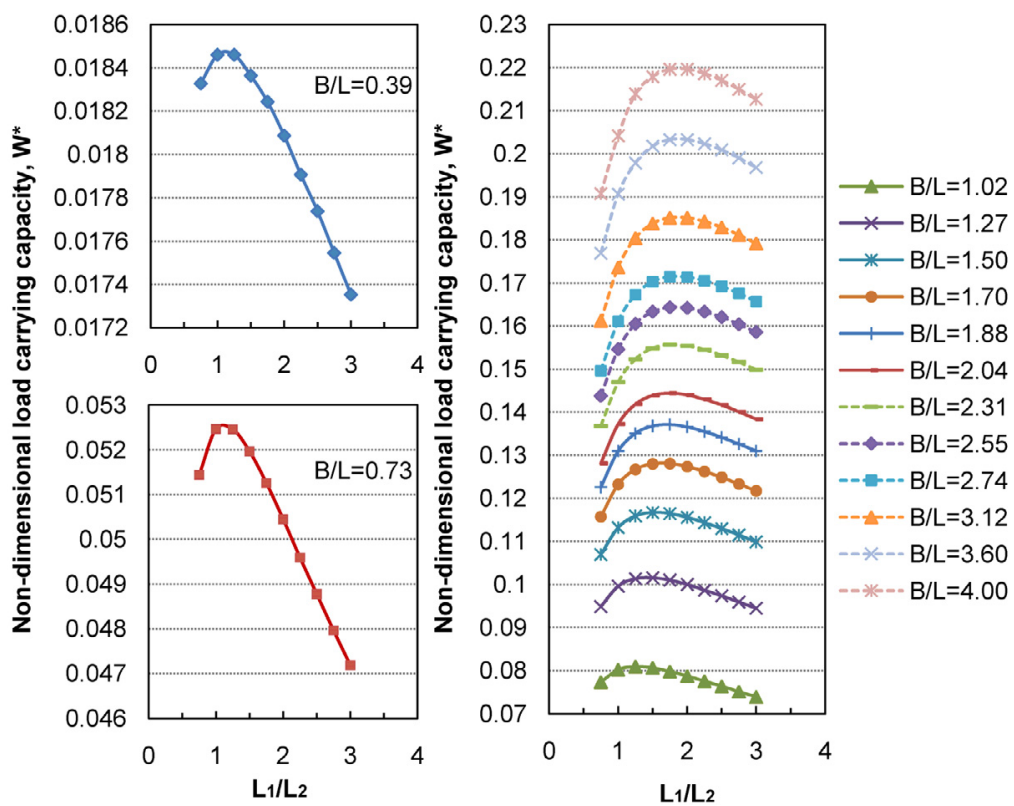

Fig. 8. Non-dimensional load carrying capacity, $W^{*}$, versus step position, $L_{1} / L_{2}$, for pads with different $B / L$ values, and $\delta^{*}=0.7$

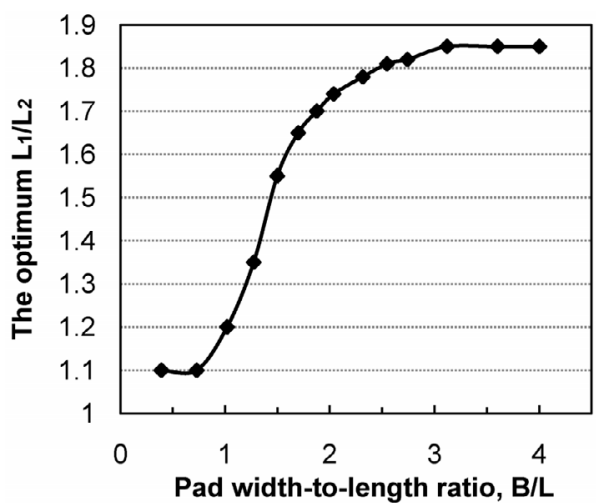

Fig. 9. Optimum $L_{1} / L_{2}$ versus pad width-to-length ratio $B / L$. $h_{2}$, an important criterion of lubrication mode, can be calculated from equation (10):

$$
h_{2}=\sqrt{\frac{W^{*} n \mu U L^{2} B}{W}}
$$

In order to ensure that the bearings operate under hydrodynamic lubrication the surface roughness values of the thrust pad and the thrust ring should satisfy the following formula [25]:

$$
\sqrt{R_{\mathrm{q}, \mathrm{p}}^{2}+R_{\mathrm{q}, \mathrm{r}}^{2}} \leqslant \frac{h_{2}}{5}
$$

where $R_{\mathrm{q}, \mathrm{p}}=$ rms (root mean square) surface roughness of the pad surface, $R_{\mathrm{q}, \mathrm{r}}=$ rms surface roughness of the ring surface. 

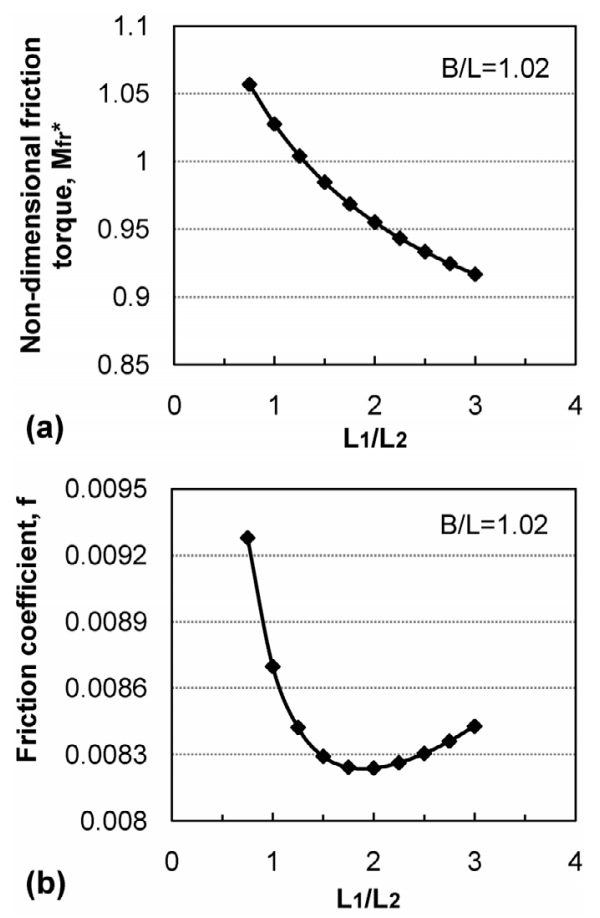

Fig. 10. (a) Non-dimensional friction torque, $M_{\mathrm{fr}}^{*}$, and (b) friction coefficient, $f$, versus $L_{1} / L_{2}$, and $\delta^{*}=0.7, B / L=1.02$.

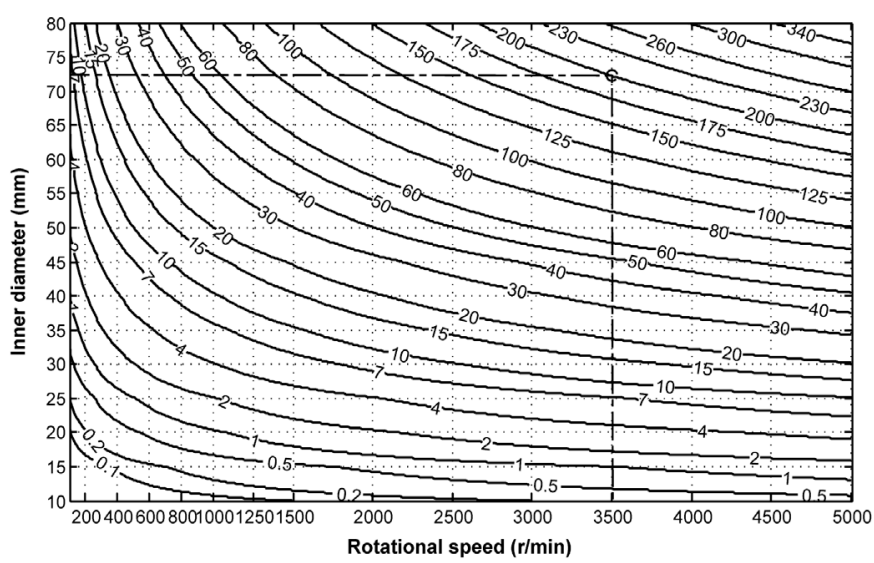

Fig. 11. Load carrying capacity map of a hydrodynamic water-lubricated thrust bearing with different dimensions and rotational speeds $\left(D_{2}=1.5 D_{1}\right)($ Unit: $\mathrm{N})$.

If the surface roughness values could not meet the design requirements, the bearing parameters should be corrected according to equation (10) and equation (11).

\section{Conclusions}

In this research, the hydrodynamic lubrication model of the water-lubricated step thrust bearing with sector pads and straight radial grooves has been fully studied using CFD method. Zwart-Gerber-Belamri model is used to simulate cavitation in the bearings. The effects of step height, step position and pad width-to-length ratio on lubrication properties are discussed. A reference to

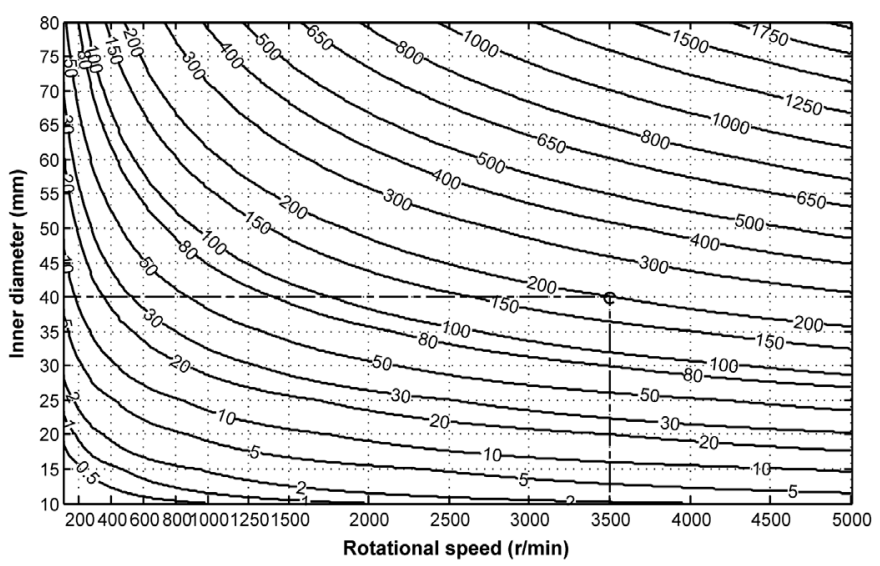

Fig. 12. Load carrying capacity map of a hydrodynamic water-lubricated thrust bearing with different dimensions and rotational speeds $\left(D_{2}=2 D_{1}\right)$ (Unit: $\left.\mathrm{N}\right)$.

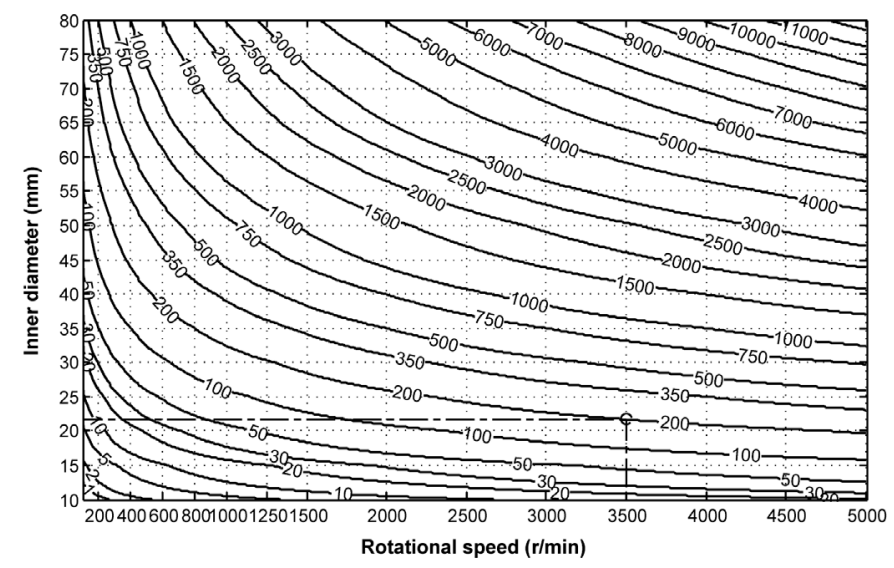

Fig. 13. Load carrying capacity map of a hydrodynamic water-lubricated thrust bearing with different dimensions and rotational speeds $\left(D_{2}=3 D_{1}\right)($ Unit: $\mathrm{N})$.

determine the bearing dimensions and a formula to check the minimum water film thickness are proposed. The main conclusions are drawn as follows:

1. The optimum non-dimensional step height, $\delta^{*}$ for the maximum load bearing capacity is about 0.68 , and the optimum $\delta^{*}$ for the minimum friction torque is about 0.8 . Considering synthetically the load carrying capacity and friction performance, the step height can be designed as $0.6 \sim 0.8$ times the minimum film thickness.

2. The optimum step position, $L_{1} / L_{2}$ resembles a sigmoid curve with the pad width-to-length ratio $B / L$. It increases rapidly from 1.2 to 1.75 when $B / L$ rises from 1 to 2 and remains 1.1 for small width pads $(B / L<1)$ and 1.85 for large width pads $(B / L>3)$.

3 . Due to the centrifugal effect in sector pads, the optimum $\delta^{*}$ and $L_{1} / L_{2}$ for pads with large width-to-length ratios are smaller than solutions of rectangular pads in the literature.

4. Initial bearing diameters can be selected from this paper. 


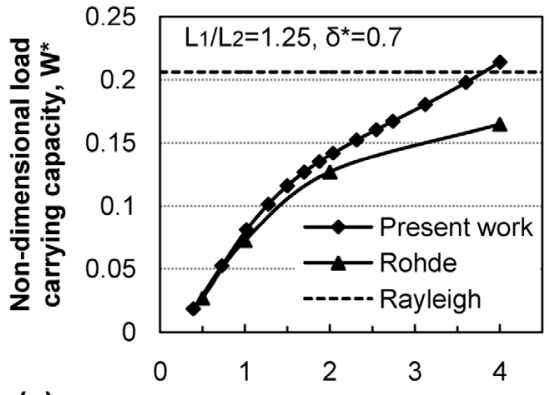

(a) Pad width-to-length ratio, B/L

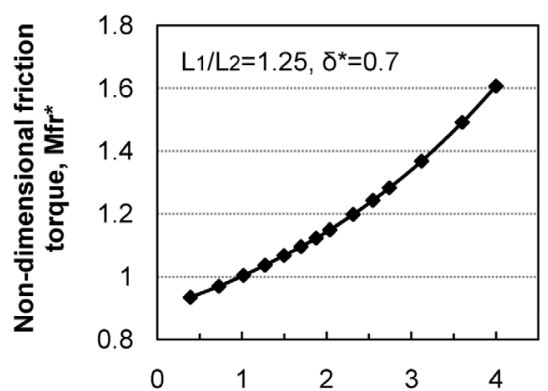

(b) Pad width-to-length ratio, B/L

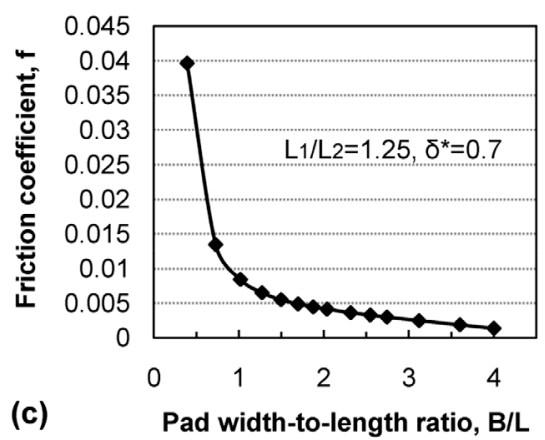

Fig. 14. Non-dimensional load carrying capacity, $W^{*}$, (b) non-dimensional friction torque, $M_{\mathrm{fr}}^{*}$, and (c) friction coefficient, $f$, versus pad width-to-length ratio $B / L$, and $\delta^{*}=0.7$, $L_{1} / L_{2}=1.25$.

5. The minimum film thickness should be checked in order to ensure that the bearings operate under hydrodynamic lubrication.

Acknowledgements. The authors thank the editor and reviewers for their helpful comments and suggestions. The support from the National Natural Science Foundation of China (Grant No. 51175341) is also gratifully acknowledged.

\section{References}

[1] R. Rahmani, A. Shirvani, H. Shirvani, Analytical analysis and optimisation of the Rayleigh step slider bearing, Tribol. Int. 42 (2009) 666-674

[2] M. Vakilian, S.A. Gandjalikhan Nassab, Z. Kheirandish, Study of inertia effect on thermohydrodynamic characteristics of Rayleigh step bearings by CFD method, Mech. Ind. 14 (2013) 275-285

[3] J. Wang, F. Yan, Q. Xue, Tribological behavior of PTFE sliding against steel in sea water, Wear 267 (2009) $1634-1641$
[4] R. Pai, D.J. Hargreaves, Water lubricated bearings, in Green Tribology, Springer, Berlin Heidelberg, 2012

[5] X. Wang, K. Koji, A. Koshi, A. Kohji, The effect of laser texturing of $\mathrm{SiC}$ surface on the critical load for the transition of water lubrication mode from hydrodynamic to mixed, Tribol. Int. 34 (2001) 703-711

[6] X. Wang, K. Kato, K. Adachi, K. Aizawa, Loads carrying capacity map for the surface texture design of SiC thrust bearing sliding in water, Tribol. Int. 36 (2003) 189-197

[7] D.L. Cabrera, N.H. Woolley, D.R. Allanson, Y.D. Tridimas, Film pressure distribution in water-lubricated rubber journal bearings, Proc. Institution Mech. Eng., Part J: J. Eng. Tribol. 219 (2005) 125-132

[8] W. Huang, Y. Xu, Y. Zheng, X. Wang, The tribological performance of $\mathrm{Ti}(\mathrm{C}, \mathrm{N})$-based cermet sliding against Si3N4 in water, Wear 270 (2011) 682-687

[9] L. Rayleigh, Notes on the theory of lubrication, Philosophical Magazine 35 (1918) 1-12

[10] F.R. Archibald, A simple hydrodynamic thrust bearing, Trans. ASME 72 (1950) 393-400

[11] C.F. Kettleborough, An electrolytic tank investigation into stepped thrust-bearings, Proceedings of the Institution of Mechanical Engineers 169 (1955) 679-688

[12] C.F. Kettlebrough, R.C.R. Johnston, An experimental investigation into stepped thrust-bearings, Proceedings of the Institution of Mechanical Engineers 170 (1956) $511-533$

[13] S.M. Rohde, Finite element optimization of finite stepped slider bearing profiles, ASLE Trans. 17 (1974) 105-110

[14] M. Dobrica, M. Fillon, Reynolds' model suitability in simulating Rayleigh step bearing thermohydrodynamic problems, Tribol. Trans. 48 (2005) 522-530

[15] P.Y.P. Chen, E.J. Hahn, Use of computational fluid dynamics in hydrodynamic lubrication, Proceedings of the Institution of Mechanical Engineers, Part J: Journal of Engineering Tribology 212 (1998) 427-436

[16] S.W. Lo, K.C. Huang, M.C. Zhou, CFD Study on oil-inwater emulsions, Tribol. Trans. 52 (2008) 66-72

[17] J.X. Zhang, C.M. Rodkiewicz, On the design of thrust bearings using a CFD technique, Tribol. Trans. 40 (1997) 403-412

[18] P. Brajdic-Mitidieri, A.D. Gosman, E. Ioannides, H.A. Spikes, CFD analysis of a low friction pocketed pad bearing, J. Tribol. 127 (2005) 803-812

[19] C.I. Papadopoulos, E.E. Efstathiou, P.G. Nikolakopoulos, L. Kaiktsis, Geometry optimization of textured threedimensional micro-thrust bearings, J. Tribol. 133 (2011) 041702

[20] X.L. Wu, Lubrication design handbook, Chemical Industry Press, Beijing, 2006

[21] ANSYS, ANSYS FLUENT, version 14.0: user manual, ANSYS, Inc., Canonsburg, USA, 2011

[22] P.J. Zwart, A.G. Gerber, T. Belamri, A two-phase flow model for predicting cavitation dynamics, in Fifth International Conference on Multiphase Flow, Yokohama, Japan, 2004

[23] Y. Rhim, J.A. Tichy, Entry flow of lubricant into a slider bearing - analysis and experiment, Tribol. Trans. 31 (1988) 351-359

[24] T.H. Yu, F. Sadeghi, Groove effects on thrust washer lubrication, J. Tribol. 123 (2001) 295

[25] B.J. Hamrock, S.R. Schmid, B.O. Jacobson, Fundamentals of fluid film lubrication, McGraw-Hill, New York, 2004 